\section{Information transmission in remote viewing experiments}

IN a recent letter to Nature ${ }^{1}$ Marks and Kammann offer criticism of the SRI experiments in 'remote viewing', the ability of certain individuals to access and describe, by means of mental processes, information blocked from ordinary perception by distance or shielding ${ }^{2-4}$. They hypothesise that the apparent success in these experiments may simply be an artefact of statements in the subjects' transcripts which provide extraneous cues useful to judges attempting to blind match transcripts to target sites. They then argue that examples from the transcripts of the first published experiment-a nine-trial series with subject Price $^{2}$-support their hypothesis. We present here experimental evidence that demonstrates that this conjecture is false.

On reading Marks and Kammann, one of us (C.T.T.), who had no connection with the original Price series, offered to independently reanalyse that series to test the validity of the Marks-Kammann hypothesis. He edited the transcripts carefully, removing all phrases suggested as potential cues by Marks and Kammann, and removing any additional phrases for which even the most remote post hoc cue argument could be made. The series was then rejudged by a new independent qualified judge (having previously shown competence in blind matching and verbal content analysis of similar materials) who was unfamiliar with the Price series. The materials turned over to the judge consisted of the newly edited transcripts presented in random order, and the list of target sites, also in random order (different from both the transcript random order and from the order of original target usage). The judge was instructed to provide, on a blind basis, a set of target/transcript correspondence ratings, which required that she visit each target site and rate transcripts to targets on a scale of 0-100 for all possible combinations, generating a $9 \times 9$ matrix. These data also yield the conventional overall measure of target/transcript correlations by indicating each first place match from the set of nine for each target.

The result of the blind matching of transcripts to target sites in the rejudging was that seven of the nine were again correctly matched. The appropriate statistic for this overall matching result is derived assuming non-independent assignment of transcripts to target sites (as in guessing the order of a random sequence of the digits one through nine, each used once) ${ }^{5}$; the result (seven out of nine correctly matched) is significant at
$P<10^{-4}$. Furthermore, the more detailed target/transcript rating matrix was analysed by an exact factorial method ${ }^{6}$; the result obtained by this analysis was significant at $P=2.2 \times 10^{-5}$. The greater significance obtained by the latter, more sensitive measure of target/transcript correlations reflected the following important fact: the judge found that, with the exception of two transcripts that did not seem to correspond to any site, the remaining seven transcripts each showed high correlation to one (correct) site and low correlation to the others. This is in direct contradiction to the implication of Marks and Kammann that little target/transcript correlation existed, and that matching is possible only on the basis of artefactual cues.

Therefore, on the basis of an independently conducted empirical test, we reject as invalid the Marks-Kammann conjecture that success in the first-published study on remote viewing is to be attributed to cueing artefacts rather than to transcript/target correlations. It is also important to note that the Marks-Kammann critique did not address the quality of the remote-viewing descriptions in the transcripts per se, but was instead limited to criticism of a particular judging procedure used to evaluate those descriptions. With regard to the descriptions themselves, we note that in the nine-transcript series in question, when the target was a boat marina the subject gave a consistent narrative that began with "What I'm looking at is a little boat jetty or boat dock along the bay. It is in a direction about like that (pointing) from here. Yeah, I see the little boats, some motor launch (sic), some little sailing ships. . . ." For a landmark Hoover Tower site, the subject summarised his impressions as "The area-I have a placeseems like it would be Hoover Tower". For a recreational swimming pool site with a $75 \mathrm{ft} \times 100 \mathrm{ft}$ rectangular pool and a $110 \mathrm{ft}$ diameter circular pool, the subject made a drawing of the target area as centred about two pools of water, which he dimensioned as a $60 \mathrm{ft} \times 89 \mathrm{ft}$ rectangular pool and a $120 \mathrm{ft}$ diameter circular pool; and so forth. Furthermore, as pointed out above, blind content analysis of the transcripts, which provides a sensitive measure of the degree of target/transcript correspondences, confirms objectively the subjective impression of above-chance correspondence that one infers from examples such as the above. With data of this quality we would argue that it is not surprising that empirical test failed to confirm the cueing-artefact hypothesis put forward by Marks and Kammann, but rather confirmed that the target/transcript matches in the first remote-viewing study are to be attributed (as originally interpreted) to the quality of the subject's descriptions themselves.

Furthermore, in the extensive SRI replication studies ${ }^{3}$, which also yielded significant results, the Marks-Kammann criticisms do not apply in principle. Target lists and transcripts were separately randomised, and transcripts were carefully checked before judging to ensure absence of any phrasing for which even a weak post hoc potential-cue argument could be made.

Given (1) the failure (by empirical test) of the cueing-artefact hypothesis to account for the success of the first-published SRI remote-viewing study ${ }^{2}$, (2) the inapplicability of this hypothesis to the later SRI replication studies ${ }^{3}$, and (3) the replication of this work in our own and other laboratories, the bulk of which is successful ${ }^{7}$, the data continue to confirm the original conclusion that remote viewing is a viable human perceptual capability.

Charles T. TART

Department of Psychology, University of California, Davis, California 95616

\section{HAROLD E. PUTHOFF RUSSELL TARG}

Radio Physical Laboratory, SRI International, Menlo Park, California 94025

\footnotetext{
1. Marks, D. \& Kammann, R. Nature 274, 680-681 (1978). 2. Targ, R. \& Puthof, H. Nature 252, 602-607 (1974)

3. Puthoff, H. \& Targ, R. Proc. IEEE 64, 329-354 (1976).

4. Targ, R. \& Puthoff, H. E. Mind-Reach (Delacorte, New York, 1977).

5. Feller, W., An Introduction to Probability Theory and Its Applications, Vol. 1, 2nd ed, 98 (Wiley, New York, Application 1957).

6. Scott, C., J. Soc. psych. Res. 46, 79-90 (1972).

7. Bisaha, J. \& Dunne, B. in Mind at Large: Institute of Electrical and Electronic Engineers Symposia on the Nature of Extrasensory Perception (eds Tart, C. T. Puthoff, H. E. \& Targ, R.) (Praeger, New York, 1979).
}

\section{Is Cassiopeia A a black hole?}

SHKLOVSKY has recently ${ }^{1}$ summarised evidence in favour of a black hole in Cas A in contrast to my arguments ${ }^{2,3}$ in favour of a binary neutron star. I defend my view here.

The fact that Cas A has not been reported as a supernova is disturbing as it has an assumed interstellar absorption $A_{\mathrm{v}}$ of $4.3 \mathrm{mag}$, and a distance of $2.8 \mathrm{kpc}$. On the other hand, Tammann ${ }^{4}$ has suggested a mean supernova rate of some $10^{-1.1 \pm 0.3} \mathrm{yr}^{-1}$ for our Galaxy; a similarly high rate is needed to account for the observed neutron stars, both pulsars and transient X-ray sources. These estimates may be reconciled if supernovae are sometimes obscured, by an adjacent dense hydrogen cloud, say, whose absorptivity is 